\title{
Polygenic risk of psychiatric disorders exhibits cross-trait associations in electronic health record data
}

Rachel L. Kember ${ }^{1,2}$; Alison K. Merikangas ${ }^{3}$, Shefali S. Verma ${ }^{1,4}$, Anurag Verma ${ }^{1,4}$, Renae Judy $^{5}$, Regeneron Genetics Center, Scott M. Damrauer ${ }^{5,6}$; Marylyn D. Ritchie ${ }^{1,4}$, Daniel J. Rader ${ }^{1,7}$; Maja Bućan ${ }^{1,8}$

1. Department of Genetics, University of Pennsylvania, Philadelphia, PA

2. MIRECC, CPL. Michael J. Crescenz VA Medical Center, Philadelphia, PA

3. Department of Biomedical and Health Informatics, Children's Hospital of Philadelphia, Philadelphia, PA

4. Institute for Biomedical Informatics, University of Pennsylvania, Philadelphia, PA, USA

5. Department of Surgery, University of Pennsylvania, Philadelphia, PA

6. Department of Surgery, CPL. Michael J. Crescenz VA Medical Center, Philadelphia, PA

7. Department of Medicine, University of Pennsylvania, Philadelphia, PA

8. Department of Psychiatry, University of Pennsylvania, Philadelphia, PA 


\section{Abstract}

Objective: Prediction of disease risk is a key component of precision medicine. Common, complex traits such as psychiatric disorders have a complex polygenic architecture making the identification of a single risk predictor difficult. Polygenic risk scores (PRS) denoting the sum of an individual's genetic liability for a disorder are a promising biomarker for psychiatric disorders, but require evaluation in a clinical setting.

Methods: We develop PRS for six psychiatric disorders (schizophrenia, bipolar disorder, major depressive disorder, cross disorder, attention-deficit/hyperactivity disorder, anorexia nervosa) and 17 non-psychiatric traits in over 10,000 individuals from the Penn Medicine Biobank with accompanying electronic health records. We perform phenomewide association analyses to test their association across disease categories.

Results: Four of the six psychiatric PRS were associated with their primary phenotypes (odds ratios between 1.2-1.6). Individuals in the highest quintile of risk had between 1.42.9 times higher odds of the disorder than the remaining $80 \%$ of individuals. Cross-trait associations were identified both within the psychiatric domain and across trait domains. PRS for coronary artery disease and years of education were significantly associated with psychiatric disorders, largely driven by an association with tobacco use disorder.

Conclusions: We demonstrate that the genetic architecture of common psychiatric disorders identified in a clinical setting confirms that which has been derived from large consortia. Even though the risk associated is low in this context, these results suggest that as identification of genetic markers proceeds, PRS is a promising approach for prediction of psychiatric disorders and associated conditions in clinical registries. 


\section{Introduction}

Psychiatric disorders are a severe global health problem, with $7.4 \%$ of all disability adjusted life years worldwide attributable to mental or behavioral health issues(1). In the United States, $18.9 \%$ of adults have a prevalent psychiatric disorder(2), and half of all US adults are estimated to experience a psychiatric disorder in their lifetime(3). Genetic variation contributing to psychiatric disorders has been identified by multiple genomewide association studies (GWAS) in large samples(4-8); however, due to their highly polygenic nature, no single variant or biomarker has been identified that reliably predicts the development of a disorder.

Polygenic risk scores (PRS) denoting an individual's genetic liability for a disorder are an emerging approach that offers the possibility of acting as a biomarker for disease(9). PRS aggregate the risk carried by multiple genetic variants, weighted by the number of alleles at each contributing polymorphic locus and the effect size of those variants in a GWAS for that disorder(10). PRS have been shown to identify individuals at increased risk for non-psychiatric disorders to an extent equivalent to large-effect monogenic mutations(11), with individuals in the highest PRS categories having as much as a 25fold increase in risk(12). In addition to their primary association with the disorder from which they were derived $(13,14)$, PRS have been used to investigate how genetic liability for a disorder is associated with intermediate phenotypes or behavioral outcomes $(15,16)$. However, nearly all psychiatric PRS have been evaluated in ascertained research cohorts, rather than tested in a clinical population. An exception to this was the finding by Zheutlin et al. (2019)(17) that a PRS for schizophrenia was robustly associated with a schizophrenia diagnosis in a health care setting, along with other medical diagnoses. The utility of other psychiatric PRS for risk prediction in clinical settings is unknown.

PRS also offer the potential to investigate the genetic overlap between disorders, by testing the association of PRS across multiple phenotypes. Psychiatric disorders share both symptomology and underlying genetic architecture $(6,18,19)$. Family members of individuals with a psychiatric disorder have an increased risk for a range of psychiatric 
disorders not limited to the specific disorder carried by their relative(20). Individuals with psychiatric disorders also have increased rates of other medical disorders(21), although the extent to which this is driven by shared genetic etiology is unclear. Assessing whether genetic liability for psychiatric disorders is disorder-specific or has broader health implications would increase our understanding of the shared genetic etiology among different disorders.

Electronic health records (EHR) provide a wealth of phenotypic information, extending across trait domains to capture a wide picture of disease burden for each individual. Linking EHR information with genotypic data allows us to investigate cross-phenotype associations of genetic risk using a phenome-wide association study (PheWAS)(22). The use of individual-level data also permits us to explore whether the cross-phenotype associations are driven by horizontal pleiotropy, in which genetic variants convey risk independently to two different phenotypes; or vertical pleiotropy, in which genetic variants convey risk to one phenotype, which in turn raises risk for a secondary phenotype(17).

To quantify the predictive value of psychiatric PRS in an EHR setting, we generated risk scores for six common psychiatric disorders (schizophrenia, bipolar disorder, major depressive disorder, cross disorder, attention-deficit/hyperactivity disorder, and anorexia nervosa) and evaluated their association with their primary phenotype in an academic biobank - the Penn Medicine Biobank (PMBB). To identify cross-trait associations and phenotypic overlap between genetic liability for psychiatric disorders, we conducted a primary PheWAS. We performed a secondary PheWAS in which we co-varied for the primary phenotype to test whether the cross-phenotypic associations remained after adjustment for the primary phenotype. Finally, we explored whether genetic risk for other common, complex traits is associated with psychiatric phenotypes in the EHR. 


\section{Subjects and Methods}

\section{Penn Medicine Biobank Cohort}

The Penn Medicine Biobank (PMBB) recruits participants through the University of Pennsylvania Health System by enrolling them at the time of a medical appointment. Patients give informed consent to participate by donating either blood or a tissue sample and allowing researchers access to their electronic health information. The study was approved by the Institutional Review Board of the University of Pennsylvania. As of November 2018, PMBB comprises 52,853 consented individuals, of whom 19,515 have been genotyped to date.

\section{Genotyping and Quality Control}

DNA extracted from the blood of 19,515 total samples were genotyped in three batches:

1) 10,867 samples on the Illumina InfiniumOmniExpress-24v1-2_A1 chip at the Regeneron Genetics Center (number of single nucleotide polymorphisms (SNPs) $=713,599$ ); 2) 5,676 samples on the Global Screening Array (GSA) V1 chip (number of SNPs $=700,078$ ) and 3) 2,972 samples on the GSA V2 chip (number of SNPs $=759,993$ ) at the Center for Applied Genomics at the Children's Hospital of Philadelphia. Each batch was quality controlled (QC) separately prior to imputation. Samples were removed if inferred sex from genotypes did not match their reported sex, or if they have a genotyping call rate $<90 \%$. SNPs were removed if call rate $<95 \%$, if they were palindromic, or if the SNPs were not present in the reference panel used for imputation. Following QC, from each batch we retained: 1) 10,506 individuals and 651,366 variants; 2) 5,660 individuals and 666,032 variants 3) 2,965 individuals and 700,984 variants.

\section{Phasing and Imputation}

Genotypes for each of the three PMBB datasets were phased (Eagle v2.3) and imputed to the 1000 Genomes reference panel (1000G Phase3 v5) using the Michigan Imputation Server(23). Following imputation, the datasets were merged, matching each position based on alleles. Genotyped variants removed during imputation were manually merged back into the final dataset. In the final merged dataset, the average quality of imputation 
was $\mathrm{R}^{2}=0.75$. Genetic ancestry was calculated from common, high-quality SNPs (MAF > 0.05 , missingness < 0.1) using the smartpca(24) module of the EIGENSOFT package. The datasets were split by genetic ancestry and QC steps were subsequently conducted independently for each population. As the calculation of PRS requires summary statistics from GWAS performed in a sample of the same ancestry as the target dataset, we retained individuals of European ancestry only for this analysis $(N=11,524)$. We removed SNPs with imputation marker $\mathrm{R}^{2}<0.7$ and minor allele frequencies $<0.01$ to retain high quality, common SNPs. Related individuals were identified using a graph-based algorithm after applying a Pi-HAT threshold of 0.25 (to identify first or second degree relatives), and the sample that was most closely related to multiple other samples was removed $(n=1,088)$. Individuals were removed if genotyped sex did not match their reported gender ( $n=85)$. Following QC, we retained 10,351 individuals. We used PLINK v1.9(25) to calculate ancestry specific principal components (PCs) to use as covariates.

\section{Polygenic risk scores}

PRS were generated using LDPred (v1.0)(26) for six psychiatric disorders and 17 other traits (see Supplemental Table 1). LDPred adjusts SNP effect sizes identified in GWAS for the effects of linkage disequilibrium (LD). To reduce the computational cost of this method, we first extracted SNPs present in the HapMap reference panel(27) from the QC'd PMBB dataset $(\mathrm{N}=1,320,405)$. LD information from the PMBB dataset was used to generate the posterior mean effect of each SNP, conditioning on a prior for genetic architecture. The prior has two parameters - the heritability of the phenotype estimated from the GWAS summary statistics, and the estimated fraction of causal markers. Due to the highly polygenic nature of the complex disorders selected for PRS, we set the parameter representing the fraction of causal variants $\rho=1$. PRS were calculated using PLINK v1.9(25) by summing all variants, weighted by the effect size of the variant following LDPred adjustment.

\section{Phenotypes}

We extracted International Classification of Diseases (ICD) ninth revision (ICD-9) and tenth revision (ICD-10) data for 52,853 individuals from the EHR, which consisted of 11.8 
million records. We filtered on encounter type to identify records representing encounters with a physician (see Supplemental Table 2 for encounters selected). We retained data for 52,354 individuals, for 9.2 million records, with 5.3 million records being ICD-9 based, and 3.9 million records being ICD-10 based. ICD-9 codes were aggregated to phecodes using the phecode ICD-9 map 1.2(28); ICD-10 codes were aggregated to phecodes using the phecode ICD-10-CM map 1.2 (beta)(29). Individuals were considered cases for the phenotype if they had at least 2 instances of the phecode on unique dates, controls if they have no instance of the phecode, and 'other/missing' if they had one instance or a related phecode. In total, 1,859 phecodes were created. The final dataset included 10,182 European individuals with complete genotype, phenotype, and covariate data.

\section{Statistical Analysis}

PRS were standardized with mean $=0$ and $S D=1$. Logistic regression was used to test for association of each psychiatric risk score with the primary phecode. The analysis was performed in $\mathrm{R}(30)$ with PRS as the independent variable and phecode as the dependent variable, and age, sex, and the first 10 ancestry-specific PCs as covariates. We performed logistic regression to estimate the odds ratio for cases when comparing the top quintile of polygenic risk to the remaining quintiles of risk.

PheWAS were performed for the psychiatric PRS. Logistic regression models with each PRS as the independent variable, phecodes as the dependent variables, and age, sex and the first $10 \mathrm{PCs}$ as covariates, were used to identify secondary phenotypic associations. Phecodes with $>100$ cases $(n=512)$ were tested. A Bonferroni-corrected phenome-wide significance threshold of $p<9.77 \times 10^{-5}$ was applied to account for multiple testing. To identify whether secondary trait associations of genetic risk were due to comorbid diagnoses of multiple phenotypes, we performed a conditional PheWAS, adjusting for the primary phenotype by including it as a covariate in the regression model. 


\section{Results}

Psychiatric phenotypes in the Penn Medicine Biobank

Of the 10,182 European ancestry individuals (men $n=6,676$, women $n=3,506$, mean age $=70.4$ ) with genotype and phenotype data in the PMBB, 3,008 (29.5\%) have at least one phecode for a psychiatric disorder. Rates of psychiatric disorders are similar between men and women with $31.3 \%$ of women in the biobank having a psychiatric disorder diagnosis $(n=1,097)$, compared to $28.6 \%$ of men $(n=1,911)$. Table 1 details the number of cases for each psychiatric disorder, grouped by parent phecode. Tobacco use disorder is the most prevalent psychiatric disorder phecode, followed by anxiety disorders and mood disorders.

Table 1. Demographics of psychiatric disorders as defined by parent phecode in the Penn Medicine Biobank

\begin{tabular}{|c|c|c|c|c|c|}
\hline \multirow[t]{2}{*}{ Phecode } & \multirow[t]{2}{*}{ Description } & \multirow{2}{*}{$\begin{array}{l}\text { \# individuals } \\
(\%)\end{array}$} & \multicolumn{2}{|l|}{ Age } & \multirow[t]{2}{*}{$\%$ Female } \\
\hline & & & Mean & SD & \\
\hline 318 & Tobacco use disorder & $1,199(11.8)$ & 69.4 & 11.1 & 29.6 \\
\hline 300 & $\begin{array}{l}\text { Anxiety, phobic and } \\
\text { dissociative disorders }\end{array}$ & $1,121(11.0)$ & 65.6 & 13.7 & 47.2 \\
\hline 296 & Mood disorders & $1,095(10.8)$ & 66.8 & 13.0 & 45.2 \\
\hline 292 & Neurological disorders & $462(4.5)$ & 73.9 & 12.0 & 31.2 \\
\hline 317 & Alcohol-related disorders & $226(2.2)$ & 66.9 & 10.2 & 16.8 \\
\hline 316 & $\begin{array}{l}\text { Substance addiction and } \\
\text { disorders }\end{array}$ & $158(1.6)$ & 62.9 & 12.6 & 29.1 \\
\hline 290 & $\begin{array}{l}\text { Delirium dementia and } \\
\text { amnestic and other } \\
\text { cognitive disorders }\end{array}$ & $129(1.3)$ & 80.7 & 8.9 & 26.4 \\
\hline 304 & Adjustment reaction & $90(0.9)$ & 66.2 & 15.5 & 47.8 \\
\hline 291 & $\begin{array}{l}\text { Other specified } \\
\text { nonpsychotic and/or } \\
\text { transient mental disorders }\end{array}$ & $73(0.7)$ & 70.8 & 12.3 & 42.5 \\
\hline 313 & $\begin{array}{l}\text { Pervasive developmental } \\
\text { disorders }\end{array}$ & $70(0.7)$ & 58.8 & 13.6 & 34.3 \\
\hline 293 & $\begin{array}{l}\text { Symptoms involving head } \\
\text { and neck }\end{array}$ & $51(0.5)$ & 70.2 & 12.2 & 33.3 \\
\hline 303 & $\begin{array}{l}\text { Psychogenic and } \\
\text { somatoform disorders }\end{array}$ & $42(0.4)$ & 61.9 & 14.5 & 42.9 \\
\hline
\end{tabular}




\begin{tabular}{l|l|l|l|l|l}
\hline 302 & $\begin{array}{l}\text { Sexual and gender identity } \\
\text { disorders }\end{array}$ & $40(0.4)$ & 67.1 & 11.6 & 10.0 \\
\hline 301 & Personality disorders & $35(0.3)$ & 68.1 & 14.6 & 40.0 \\
\hline 295 & $\begin{array}{l}\text { Schizophrenia and other } \\
\text { psychotic disorders }\end{array}$ & $33(0.3)$ & 67.2 & 13.9 & 27.3 \\
\hline 306 & Other mental disorder & $32(0.3)$ & 65.0 & 13.0 & 59.4 \\
\hline 297 & $\begin{array}{l}\text { Suicidal ideation or } \\
\text { attempt }\end{array}$ & $18(0.2)$ & 61.2 & 14.5 & 38.9 \\
\hline 305.2 & Eating disorders & $13(0.1)$ & 52.3 & 18.8 & 53.8 \\
\hline 315 & $\begin{array}{l}\text { Developmental delays and } \\
\text { disorders }\end{array}$ & $8(0.1)$ & 77.3 & 10.2 & 37.5 \\
\hline 312 & Conduct disorders & $4(0.03)$ & 69.2 & 5.6 & 25.0 \\
\hline- & Any psychiatric disorder & $3,008(29.5)$ & 68.8 & 12.8 & 36.5 \\
\hline
\end{tabular}

Primary associations of psychiatric PRS

We generated PRSs for common psychiatric disorders based on publicly available summary statistics from genome-wide association studies conducted by the Psychiatric Genomics Consortium(31) (PGC, See Supplemental Table 1 for individual references). We calculated six psychiatric PRSs, including schizophrenia (SCZ), bipolar disorder (BD), major depressive disorder (MDD), cross disorder (CROSS), attention-deficit/hyperactivity disorder (ADHD), and anorexia nervosa (AN) and tested their association with their respective primary phenotypes (Supplemental Table 3 ). $\mathrm{PRS}_{\mathrm{Scz}}$ was significantly associated with schizophrenia (phecode 295, $\mathrm{N}$ cases $=33$, OR=1.46, 95\% Cl 1.01-2.11, $p=0.046$ ). $P R S_{B D}$ was significantly associated with bipolar disorder (phecode 296.1, N cases $\left.=96, \mathrm{OR}=1.58,95 \% \mathrm{Cl} 1.27-1.95, \mathrm{p}=3.9 \times 10^{-5}\right)$. $P R S_{M D D}$ was significantly associated with major depressive disorder (phecode 296.22, $\mathrm{N}$ cases $=446, \mathrm{OR}=1.22,95 \% \mathrm{Cl} 1.10$ $\left.1.34, p=1.0 \times 10^{-4}\right)$. $P R S_{\text {CROSS }}$ was significantly associated with a composite phenotype $(\mathrm{N}$ cases $=578, \mathrm{OR}=1.17,95 \% \mathrm{Cl} 1.06-1.29, \mathrm{p}=0.001)$ comprised of autism spectrum disorders (phecode 313.3) or ADHD (phecode 313.1) or bipolar disorder (phecode 296.1) or major depressive disorder (phecode 296.22) or schizophrenia (phecode 295). Neither PRS $_{A D H D}$ nor $P_{R S_{A N}}$ were significantly associated with their primary phenotypes (ADHD: phecode 313.1, AN: phecode 305.2). 
Case prevalence per PRS quintile was calculated for each phenotype (Figure 1, Supplemental Table 4). Absolute risk for patients in the top quintile of PRSscz was $0.84 \%$ $(0.37 \%$ in the remaining $80 \%)$, with a corresponding odds ratio of $2.86(95 \% \mathrm{Cl} 1.38-5.97$, $\mathrm{p}=0.005)$. For $\mathrm{PRS}_{\mathrm{BD}}$ absolute risk for the top quintile was $2.36 \%$ (1.09\% in the remaining $80 \%, \mathrm{OR}=2.21,95 \% \mathrm{Cl}$ 1.42-3.44, $\mathrm{p}=0.0004)$; for $\mathrm{PRS}_{\mathrm{MDD}}$ absolute risk in the top quintile was $7.69 \%$ (5.79\% in the remaining $80 \%$, OR=1.42, 95\% Cl 1.14-1.77, $p=0.002)$. The top quintiles of $P R S_{C R O S S}, \mathrm{PRS}_{\mathrm{ADHD}}$ and $\mathrm{PRS}_{\mathrm{AN}}$ showed no difference in absolute risk compared to the remaining $80 \%$.

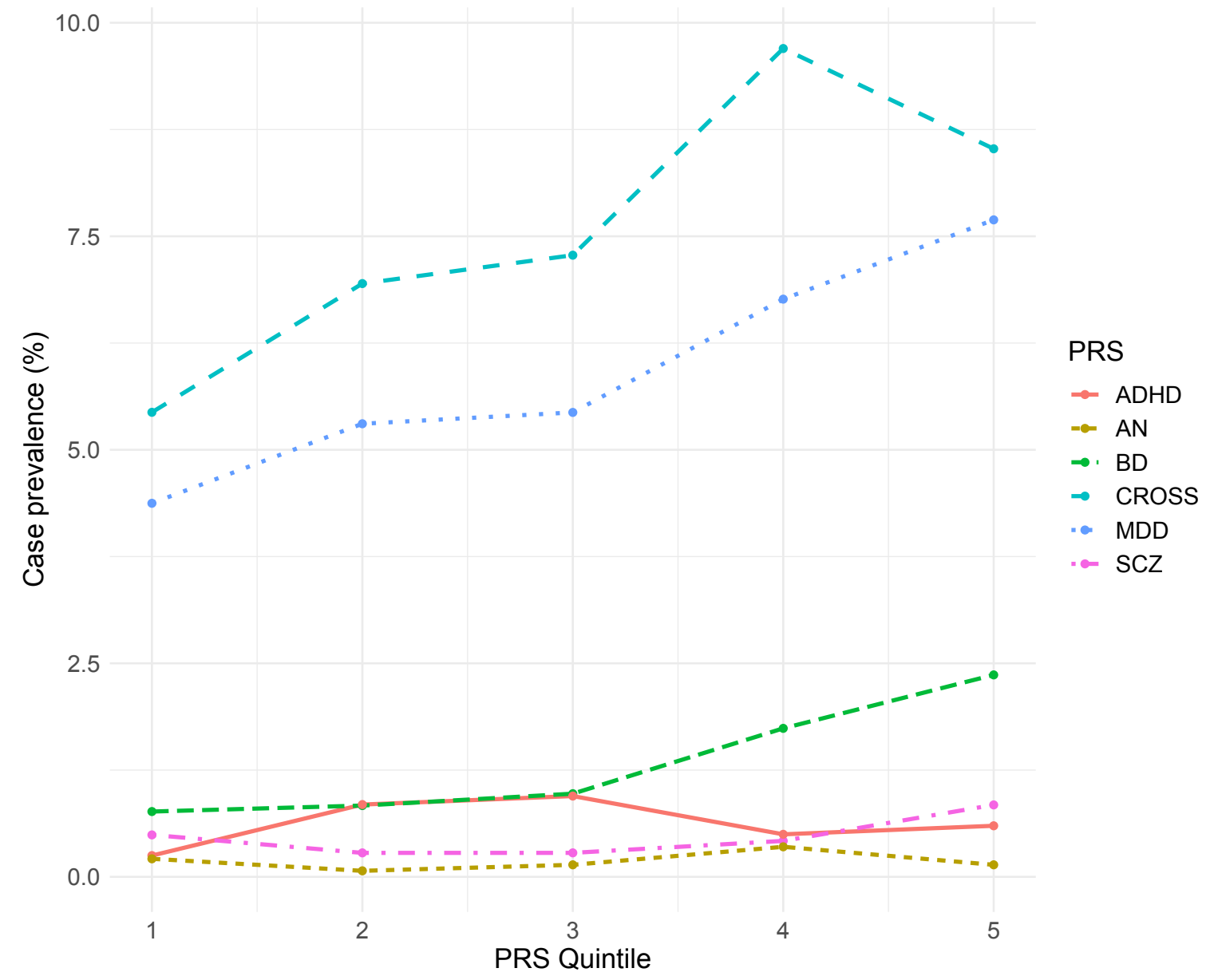

Figure 1. Case prevalence (\%) by PRS quintile for schizophrenia (SCZ, phecode 295), bipolar disorder (BD, phecode 296.1), major depressive disorder (MDD, phecode 296.22), cross disorder (CROSS, phecodes 295, 296.1, 296.22, 313.1, 313.3), attention deficit/hyperactivity disorder (ADHD, phecode 313.1), and anorexia nervosa (AN, phecode 305.2) PRS. 
Association of polygenic risk scores with burden of psychiatric disorders

More than one-third $(39.4 \%)$ of individuals $(n=1,185)$ with a psychiatric disorder have multiple such diagnoses (Supplemental Figure 1A), as defined by the number of diagnoses for parent psychiatric phecodes within an individual. We explored the association of the psychiatric PRSs with the burden of psychiatric disorder in cases with at least one psychiatric disorder phecode. $\mathrm{PRS}_{\mathrm{scz}}$ (beta=0.043, p=0.024), PRS (beta=0.060, $p=0.003$ ), and PRS CRoss (beta=0.066, $p=8.98 \times 10^{-4}$ ) were significantly associated with an increased burden of psychiatric disorder phenotypes (Supplemental Table 5, Supplemental Figure 1B).

Phenome-wide analysis of psychiatric polygenic risk scores show cross-trait associations

To identify secondary phenotypes associated with the psychiatric PRS we conducted a PheWAS for all phecodes with $>100$ cases $(n=512)$, applying a Bonferroni multiple testing correction $\left(p<9.77 \times 10^{-5}\right)$ (Figure 2). The strongest phenotypic associations for $\mathrm{PRS}_{\mathrm{BD}}$, $\mathrm{PRS}_{\mathrm{MDD}}, \mathrm{PRS}_{\mathrm{CROSS}}$ and PRS $\mathrm{ADHD}$ were in the psychiatric disorders domain, whereas the strongest associations for $\mathrm{PRS}_{\mathrm{SCZ}}$ and $\mathrm{PRS}_{\mathrm{AN}}$ were in the genitourinary and endocrine/metabolic domains, respectively. PRSscz was significantly positively associated with "urinary system disorders" $\left(\mathrm{OR}=1.17, \mathrm{p}=2.5 \times 10^{-5}\right)$ and "anxiety disorder" $\left(O R=1.16, p=8.1 \times 10^{-5}\right)$, in addition to 11 traits at a less stringent false-discovery rate (FDR) p-value <0.05 (Supplemental Table 6). $\mathrm{PRS}_{\mathrm{BD}}$ was associated with "mood disorders" ( $\left.\mathrm{OR}=1.19, \mathrm{p}=9.3 \times 10^{-7}\right)$ and "anxiety, phobic and dissociative disorders" $\left(\mathrm{OR}=1.17, \mathrm{p}=8.7 \times 10^{-6}\right)$, with associations for an additional 6 traits at FDR $\mathrm{p}<0.05$ (Supplemental Table 7). PRS $\operatorname{MDD}$ was associated with "anxiety, phobic and dissociative disorders" $\left(\mathrm{OR}=1.23, \mathrm{p}=7.2 \times 10^{-10}\right)$, "mood disorders" $\left(\mathrm{OR}=1.21, \mathrm{p}=1.0 \times 10^{-8}\right)$, "tobacco use disorder" $\left(\mathrm{OR}=1.14, \mathrm{p}=2.5 \times 10^{-5}\right)$, and "chronic airway obstruction" $(\mathrm{OR}=1.14$, $\mathrm{p}=4.4 \times 10^{-5}$ ), as well as 8 other traits at FDR $\mathrm{p}<0.05$ (Supplemental Table 8). PRScross was associated with "mood disorders" $\left(O R=1.17, p=2.6 \times 10^{-5}\right)$ with an additional trait associated at FDR $p<0.05$ (Supplemental Table 9). PRS ADHD was positively associated with "tobacco use disorder" $\left(\mathrm{OR}=1.27, \mathrm{p}=8.4 \times 10^{-14}\right)$, "chronic airway obstruction" 
$\left(\mathrm{OR}=1.20, \mathrm{p}=2.2 \times 10^{-8}\right)$ and "type 2 diabetes" $\left(\mathrm{OR}=1.10, \mathrm{p}=9.5 \times 10^{-5}\right)$, and negatively associated with "benign neoplasm of skin" ( $\left.O R=0.85, p=8.6 \times 10^{-9}\right)$ and "myopia" $\left(\mathrm{OR}=0.74, \mathrm{p}=2.4 \times 10^{-5}\right)$, in addition to 22 traits at FDR $p<0.05$ (Supplemental Table 10). PRS $_{\mathrm{AN}}$ was negatively associated with "overweight, obesity and other hyperalimentation" $\left(\mathrm{OR}=0.89, \mathrm{p}=2.7 \times 10^{-6}\right)$, and "obesity" at FDR $\mathrm{p}<0.05$ (Supplemental Table 11).

Using an FDR threshold of $p<0.05$ to select significant phenotypic associations, we explored the phenotypic overlap between the psychiatric PRS (Supplemental Table 12). Almost all of the shared phenotypes were in the psychiatric disorders domain, reflecting the main associations identified above. The highest overlap was between $P R S_{B D}$ and $\mathrm{PRS}_{\mathrm{MDD}}$, with five mental disorder phenotypes (Mood disorders; Depression; Anxiety, phobic and dissociative disorders; Anxiety disorder; Generalized anxiety disorder) associated with genetic liability for both disorders. PRS $S_{M D D}$ also shared four phenotypic associations (Anxiety, phobic and dissociative disorders; Anxiety disorder; Tobacco use disorder; Chronic airway obstruction) with PRS $\mathrm{ADHD}_{\text {. }}$ PRS $\mathrm{AN}_{\mathrm{AN}}$ had the lowest phenotypic overlap with other psychiatric PRS. Both $P R S_{A N}$ and PRS $S_{A D H D}$ were associated with "overweight, obesity and other hyperalimentation", although the associations were in the opposite direction.

To identify whether secondary trait associations of genetic risk were due to comorbid diagnoses of multiple phenotypes, we re-ran the phenome-wide association analyses with the addition of a covariate for the corresponding phenotype (Supplemental Tables 6-11). The majority of phenotypic associations showed little change, with the secondary associations identified for PRS $\mathrm{SCZ}, \mathrm{PRS} \mathrm{B}_{\mathrm{BD}}, \mathrm{PRS}_{\mathrm{ADHD}}$ and $\mathrm{PRS}_{\mathrm{AN}}$ remaining significant. The association between PRS $\mathrm{MDD}_{\text {and }}$ abacco use disorder was reduced and no longer survived Bonferroni correction, although the effect size was similar in magnitude $\left(O R=1.13, p=1.2 \times 10^{-4}\right)$. $P R S_{C R O S s}$ was no longer significantly associated with mood disorders after Bonferroni correction (OR=1.15, $\mathrm{p}=0.003)$. 

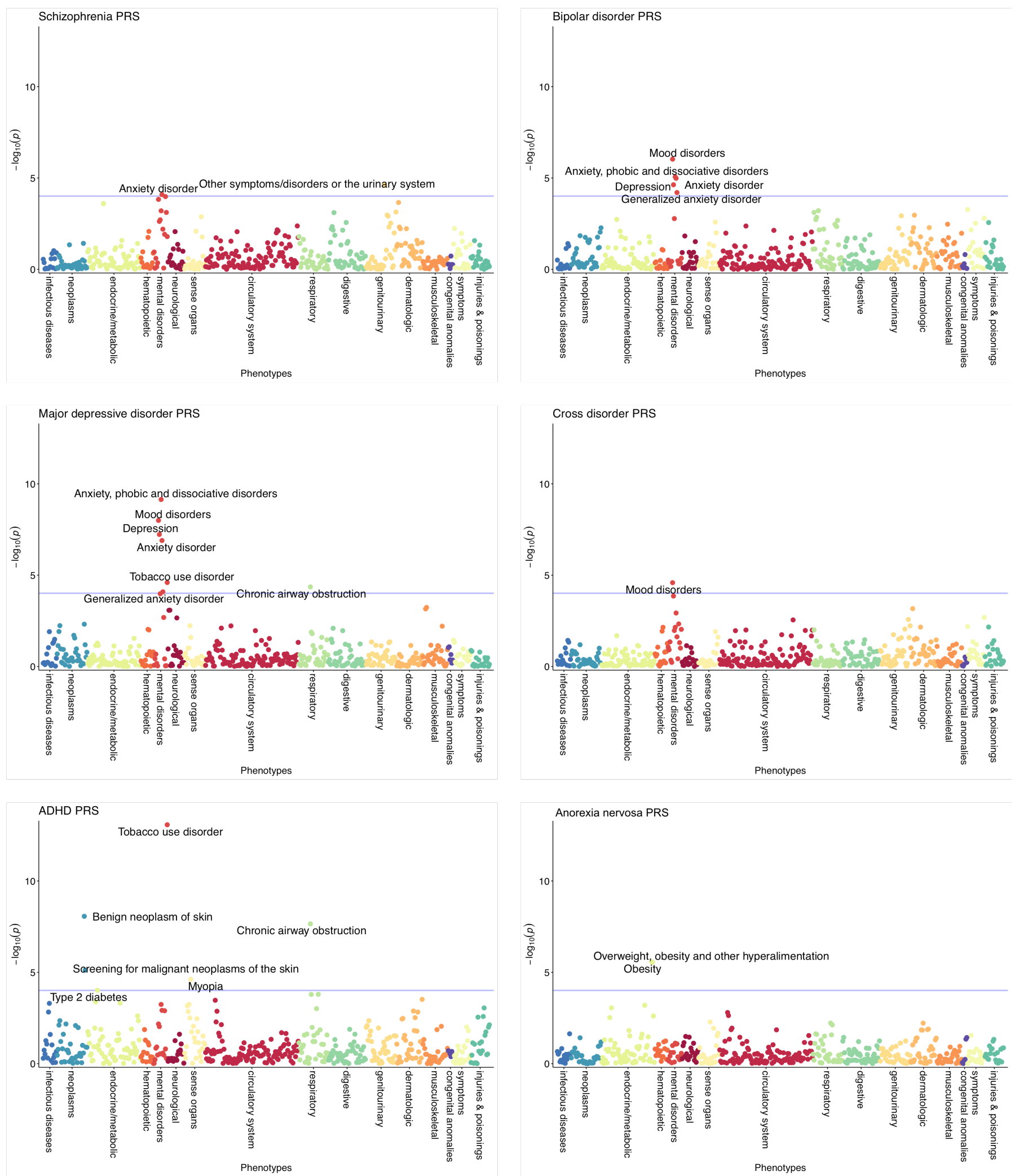

Figure 2: Phenome-wide association of psychiatric polygenic risk scores. A phenomewide association plot is shown for each psychiatric PRS (schizophrenia, bipolar disorder, 
major depressive disorder, cross disorder, attention deficit/hyperactivity disorder, and anorexia nervosa). Phenotypes with $>100$ cases were tested $(n=512)$ in 10,182 individuals from the Penn Medicine Biobank. Phenotypes are grouped by trait domain along the horizontal axis, the significance of association between the PRS and phenotype is shown on the vertical axis (- $\log _{10} \mathrm{p}$; two-tailed). The blue line indicates phenome-wide significance following Bonferroni correction $\left(p<9.77 \times 10^{-5}\right)$. Phenotypes reaching phenome-wide significance are labelled.

PRS for other traits predict psychiatric phenotypes

Psychiatric disorders are often comorbid with other medical disorders. We therefore generated PRS for an additional 17 traits and disorders previously associated with psychiatric phenotypes, and explored their correlational structure (Figure 3A, Supplemental Table 13). PRS for psychiatric disorders were significantly positively correlated with each other, with the strongest associations being between

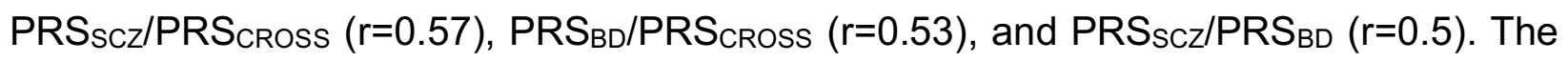
psychiatric PRSs were also positively correlated with PRS for diabetes ( $r=0.06$ to 0.24 ), former smoking ( $r=0.08$ to 0.28 ) and total cholesterol ( $r=0.0$ to 0.23 ), and negatively correlated with birthweight ( $r=-0.02$ to -0.11 ). Years of education PRS was positively correlated with $\mathrm{PRS}_{S C Z}(r=0.12), \mathrm{PRS}_{\mathrm{BD}}(r=0.17), \mathrm{PRS}_{\mathrm{AN}}(r=0.14)$ and PRS $\mathrm{PROSS}_{(}(r=0.18)$, and negatively correlated with PRS MDD $(r=-0.09)$ and PRS ADHD $_{(r=-0.21)}$. Coronary artery disease PRS was positively correlated with $\mathrm{PRS}_{\mathrm{ADHD}}(\mathrm{r}=0.08)$, and negatively correlated with PRS CROSS $_{(r=-0.11), \text { PRS }_{A N}(r=-0.04) \text {, and PRS }}$ BD $(r=-0.06)$.

To identify whether genetic liability for these traits was associated with a broad definition of psychiatric disorder, we identified all individuals with at least one phecode for any psychiatric disorder as defined in Table $1(\mathrm{~N}=3,008)$. Of the 23 PRS tested, 6 were associated with risk for psychiatric disorder, broadly defined, at a Bonferroni-corrected pvalue $<0.002$ (Figure 3B and Supplemental Table 14). The strongest association was with $\mathrm{PRS}_{\mathrm{MDD}}\left(\mathrm{OR}=1.16, \mathrm{p}=2.6 \times 10^{-10}\right)$ followed by $\mathrm{PRS}_{\mathrm{ADHD}}\left(\mathrm{OR}=1.12, \mathrm{p}=2.3 \times 10^{-6}\right)$. PRS $\mathrm{scz}$ and PRS $\mathrm{BD}_{\mathrm{BD}}$ were also significantly associated with having a psychiatric disorder (SCZ: 
$\left.\mathrm{OR}=1.09, p=6.4 \times 10^{-4} ; \mathrm{BD}: \mathrm{OR}=1.09, \mathrm{p}=7.6 \times 10^{-4}\right)$. Two non-psychiatric PRS were associated with psychiatric disorder; PRS for coronary artery disease (CAD) was positively associated with psychiatric disorder $\left(O R=1.10, p=5.0 \times 10^{-4}\right)$, and $P R S$ for years of education (EduYears) was negatively associated with psychiatric disorder ( $O R=0.91$, $\left.p=4.1 \times 10^{-4}\right)$. We conducted a secondary association test within psychiatric disorder

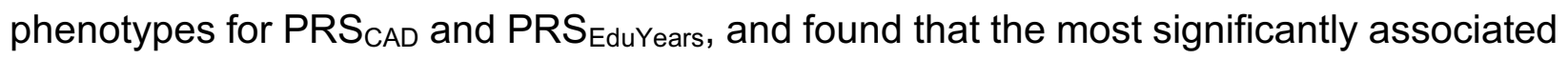
psychiatric disorder for both PRS was "tobacco use disorder" (Supplemental Table 15). 


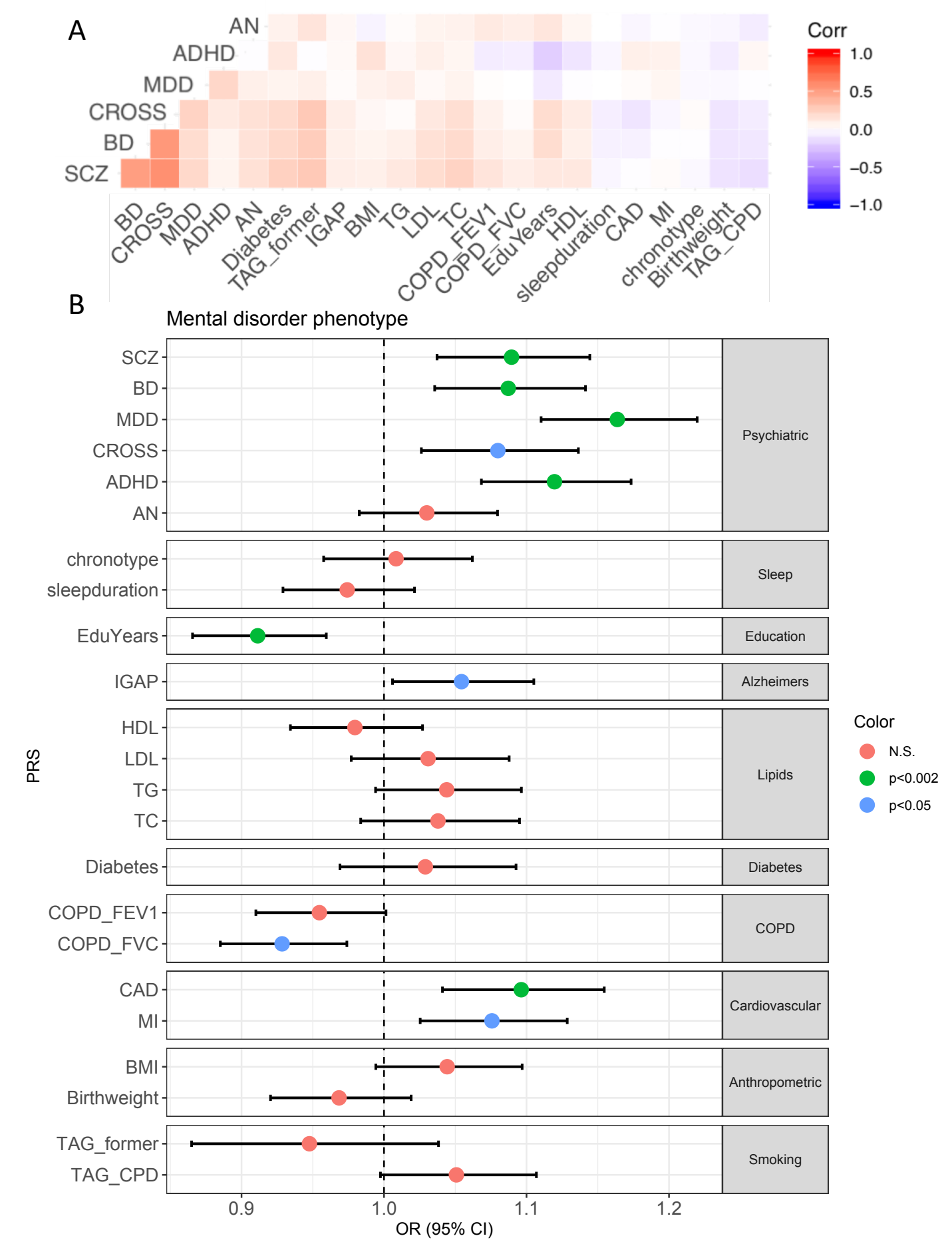

Figure 3. Polygenic risk for other traits. SCZ=schizophrenia, BD=bipolar disorder, $M D D=$ major depressive disorder, $\quad$ CROSS=cross disorder, $A D H D=$ attention deficit/hyperactivity disorder, AN=anorexia nervosa, chronotype=chronotype, 
sleepduration=sleep duration, EduYears=years of education, IGAP=Alzheimer's, HDL=high-density lipoproteins, LDL=low-density lipoproteins, TG=triglycerides, TC=total cholesterol, Diabetes=type 2 diabetes, COPD_FEV1=chronic obstructive pulmonary disease forced expiratory volume, COPD_FVC= chronic obstructive pulmonary disease forced vital capacity, $\mathrm{CAD}=$ coronary artery disease, $\mathrm{MI}=$ myocardial infarction, $\mathrm{BMI}=$ body mass index, Birthweight=birthweight, TAG_former=current vs. former smoker, TAG_CPD=cigarettes per day. A: Correlational structure between psychiatric PRS and PRS for other traits. Positive correlations are denoted in red, negative correlations are denoted in blue, white squares are non-significant. B: Association between all PRS and psychiatric disorder, broadly defined. PRS are grouped by trait domain. Odds ratios and 95\% confidence intervals are shown for the association between each PRS and psychiatric disorder. Psychiatric disorder was defined as an individual having any psychiatric disorder phecode. 


\section{Discussion}

Here, we present our examination of genetic liability for six psychiatric disorders with phenotypes derived from EHR diagnosis codes in 10,182 individuals from the Penn Medicine Biobank. We identify several important findings. We establish the utility of multiple psychiatric PRS in a clinical setting, showing that PRS for schizophrenia, bipolar disorder, major depressive disorder, and cross disorder are associated with their expected diagnoses in EHR. We identify cross-trait associations of psychiatric PRS mostly within the psychiatric domain, indicating overlap in the underlying genetic risk for these conditions. We identify genetic liability for two non-psychiatric disorders that increase risk of a psychiatric diagnosis.

Of the six psychiatric disorders tested, we found a significant association of genetic liability for schizophrenia, bipolar disorder, major depressive disorder, and cross disorder with their primary phenotypes, but not for attention-deficit/hyperactivity disorder and anorexia nervosa. The number of cases of ADHD and eating disorders (ED) is low in the PMBB (ADHD $n=63$, ED $n=13$ ), providing limited power to find a significant association. However, despite the small number of schizophrenia cases $(n=33)$, we were able to identify a significant association between $\mathrm{PRS}_{\mathrm{scz}}$ and schizophrenia diagnosis. It is worth noting that the schizophrenia GWAS ( $n=36,989$ cases) is nearly twice the sample size of the ADHD GWAS ( $n=20,183$ cases), and ten times the sample size of the anorexia nervosa GWAS ( $n=3,495$ cases). Misclassification or under diagnosis of ADHD and ED could also be responsible for our lack of association. Cross-trait analyses for PRS $\mathrm{ADHD}_{\mathrm{A}}$ identified an association with tobacco use disorder, which is known to be highly prevalent in individuals with $\operatorname{ADHD}(32)$, suggesting that we are capturing some of the genetic risk for the disorder. Likewise, cross-trait analyses for $\mathrm{PRS}_{\mathrm{AN}}$ showed a negative association with obesity, which aligns with a recent study where the authors suggest the increased liability to AN in females may be due to sex-specific anthropometric and metabolic genetic factors, specifically body fat percentage(33). In our study it is unclear as whether individuals with high $\mathrm{PRS}_{\mathrm{AN}}$ have a lower propensity for obesity in the absence of an eating disorder, or whether the result is confounded by the presence of an undiagnosed 
eating disorder. However, this finding also suggests that we are capturing at least some of the true genetic liability for disorder.

The effect sizes of association with primary phenotypes when comparing the top quintile of psychiatric PRS to the remaining $80 \%$ (odds ratios between 1.42-2.86) are in line with previous studies of PRS in clinical settings, where non-psychiatric phenotypes including cardiovascular disease, atrial fibrillation, and type 2 diabetes have shown effect sizes in the range of 2.33-2.55(11). The schizophrenia PRS had an effect size of 2.3 in a previous study(17), compared to an effect size of 2.86 here, although our confidence intervals are larger due to the smaller sample size. Despite these medium effect sizes, the significant association of psychiatric PRS with their primary phenotypes does not provide the ability to predict the development of disorder in a naïve patient. For instance, an odds ratio for absolute risk of bipolar disorder in the top quintile of $\mathrm{PRS}_{\mathrm{BD}}$ vs. the other $80 \%$ of 2.2 translates to a difference in disorder prevalence of only $1.27 \%$. Psychiatric PRS will have to explain much more phenotypic variance and differentiate effectively cases from controls before they will be considered clinically useful. We expect that results from future GWAS will continue to improve our estimates of genetic risk, such that we can begin to consider the use of PRS as a clinical risk predictor.

PheWAS of psychiatric PRS revealed cross-trait associations, many of which were within

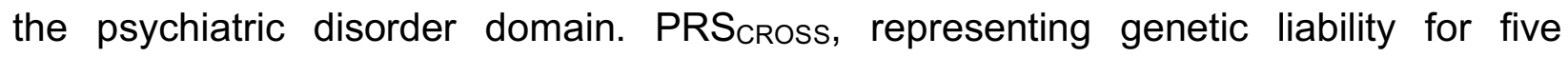
psychiatric disorders, was associated with mood disorder in a clinical setting. PRSscz, $\mathrm{PRS}_{\mathrm{BD}}, \mathrm{PRS} \mathrm{S}_{\mathrm{MDD}}$, and $\mathrm{PRS}_{\mathrm{ADHD}}$ were all associated with additional psychiatric phenotypes, suggesting a broader effect of genetic liability for these disorders. Of these, PRSscz, $\mathrm{PRS}_{\mathrm{BD}}$, and PRS $\mathrm{PROss}$ were also associated with an increased burden of psychiatric diagnoses, suggesting that higher genetic risk for these disorders contributes in a nondisorder specific manner to disorder load. These associations could be due to genetic liability for psychiatric disorder going beyond traditional diagnostic boundaries, or imprecision in the assignment of ICD codes at time of diagnosis. PRS ADHD $_{\text {exhibited the }}$ most non-psychiatric phenotypic associations, including a positive association with type 2 diabetes. A positive genetic correlation between ADHD and type 2 diabetes was 
identified in the GWAS(7) that provided the summary statistics for the PRS; we confirm that this association can be replicated using individual level data.

Previous work showing cross-phenotype associations for PRSscz identified many more phenotypic associations than we find here(17), likely due to the substantially larger sample size. We replicate two of these associations - anxiety disorder and urinary system disorders. Anxiety disorders were the most significant cross-phenotype association identified in Zheutlin et al. (2019), and have previously been shown to share genetic liability with schizophrenia(34). In addition, anxiety symptoms are often reported in the prodromal phase of psychoses or schizophrenia, before acute episodes, as well as being common among people with the disorder(35). We replicate the association with urinary disorders despite the fact that it was not one of the strongest associations in the prior study. Furthermore, this association remained significant in a sensitivity analysis, suggesting that it is not driven by the diagnosis of schizophrenia. Using a less stringent p-value threshold, we find many of the other phenotypic associations identified in the prior study, suggesting that these findings may be replicable across datasets.

The structure of genetic correlations we identify using individual-level PRS is largely consistent with prior genetic correlation findings for each of the psychiatric disorders. Within the psychiatric domain, PRS correlations reflect similar results to prior work showing that schizophrenia and bipolar disorder have substantial genetic overlap, and that there is moderate overlap between MDD and SCZ, BD, and ADHD(36). We also find that a genetic liability for specific disorders is associated with risk for any psychiatric disorder, defined as any diagnosis within the psychiatric disorder domain. Interestingly, we also find significant associations for genetic liability of coronary artery disease and years of education with risk for psychiatric disorder. Both of these associations appear to be driven by an association with tobacco use disorder, the largest mental disorder phenotypic group in our dataset. Despite the overall decline in tobacco use in the US over the past few decades, there are still health disparities in use and the health consequences of smoking among different social strata, including race, ethnicity, educational level, socioeconomic status, and geographic region. Notably, people with low SES are reported 
to smoke cigarettes more heavily, and to suffer from more diseases caused by smoking then do people from higher SES brackets. Moreover, the rate of smoking is higher among people who have a mental illness than those who do not $(32.0 \%$ vs $23.2 \%)(37)$.

Our study has several limitations. The initial population available for this study contained twice as many men as women, is on average older than the general population, and a large proportion had cardiovascular disease due to the initial PMBB recruitment strategy that focused on patients with such disease (though it now recruits broadly from the health system). As researchers using EHR continue to expand and recruit more broadly from the population they serve, we expect that biobanks will become more reflective of the clinical population. Importantly, we limited our analysis to European ancestry individuals only due to the lack of GWAS summary statistics for other populations. As this area of research continues to develop, it will be critical for GWAS to be conducted in diverse ancestries to allow the clinical implications of genetic risk to be tested across populations.

We show that genetic liability for schizophrenia, bipolar disorder, major depressive disorder, and cross disorder is associated with psychiatric phenotypes derived from EHR, with effect sizes comparable to other clinical risk factors. We further conclude that genetic liability for these disorders is broadly associated with multiple psychiatric phenotypes in addition to phenotypes in other disease domains. These pleiotropic effects support evidence of an extensive shared genetic architecture among common traits. As identification of genetic markers associated with psychiatric disorders continues to improve, our results provide evidence that the application of PRS is a promising future approach for risk prediction of psychiatric disorders and associated conditions in clinical settings. 


\section{References}

1. Murray CJL, Vos T, Lozano R, Naghavi M, Flaxman AD, Michaud C, et al.

Disability-adjusted life years (DALYs) for 291 diseases and injuries in 21 regions, 1990-2010: a systematic analysis for the Global Burden of Disease Study 2010. Lancet (London, England) [Internet]. 2012 Dec 15;380(9859):2197-223. Available from: http://www.ncbi.nlm.nih.gov/pubmed/23245608

2. Substance Abuse and Mental Health Services Administration. Key substance use and mental health indicators in the United States: Results from the 2017 National Survey on Drug Use and Health (HHS Publication No. SMA 18-5068, NSUDH Series H-53) [Internet]. Rockville, MD; 2018. Available from: https://www.samhsa.gov/data/sites/default/files/cbhsqreports/NSDUHFFR2017/NSDUHFFR2017.pdf

3. Kessler RC, Angermeyer M, Anthony JC, DE Graaf R, Demyttenaere K, Gasquet I, et al. Lifetime prevalence and age-of-onset distributions of mental disorders in the World Health Organization's World Mental Health Survey Initiative. World Psychiatry [Internet]. 2007 Oct;6(3):168-76. Available from:

http://www.ncbi.nlm.nih.gov/pubmed/18188442

4. Howard DM, Adams MJ, Clarke T-K, Hafferty JD, Gibson J, Shirali M, et al. Genome-wide meta-analysis of depression identifies 102 independent variants and highlights the importance of the prefrontal brain regions. Nat Neurosci [Internet]. 2019 Mar 4;22(3):343-52. Available from: http://www.nature.com/articles/s41593-018-0326-7

5. Schizophrenia Working Group of the Psychiatric Genomics Consortium. Biological insights from 108 schizophrenia-associated genetic loci. Nature [Internet]. 2014 Jul 24;511(7510):421-7. Available from: http://www.ncbi.nlm.nih.gov/pubmed/25056061

6. Cross-Disorder Group of the Psychiatric Genomics Consortium. Identification of risk loci with shared effects on five major psychiatric disorders: a genome-wide analysis. Lancet (London, England) [Internet]. 2013 Apr 20;381(9875):1371-9. Available from: http://www.ncbi.nlm.nih.gov/pubmed/23453885 
7. Demontis D, Walters RK, Martin J, Mattheisen M, Als TD, Agerbo E, et al. Discovery of the first genome-wide significant risk loci for attention deficit/hyperactivity disorder. Nat Genet [Internet]. 2019;51(1):63-75. Available from: http://www.ncbi.nlm.nih.gov/pubmed/30478444

8. Sullivan PF, Agrawal A, Bulik CM, Andreassen OA, Børglum AD, Breen G, et al. Psychiatric Genomics: An Update and an Agenda. Am J Psychiatry [Internet]. 2017 Oct 3;175(1):15-27. Available from: https://doi.org/10.1176/appi.ajp.2017.17030283

9. Martin AR, Daly MJ, Robinson EB, Hyman SE, Neale BM. Predicting Polygenic Risk of Psychiatric Disorders. Biol Psychiatry [Internet]. 2019; Available from: https://doi.org/10.1016/j.biopsych.2018.12.015

10. Purcell SM, Wray NR, Stone JL, Visscher PM, O'Donovan MC, Sullivan PF, et al. Common polygenic variation contributes to risk of schizophrenia and bipolar disorder. Nature. 2009 Aug 6;460(7256):748-52.

11. Khera A V, Chaffin M, Aragam KG, Haas ME, Roselli C, Choi SH, et al. Genomewide polygenic scores for common diseases identify individuals with risk equivalent to monogenic mutations. Nat Genet [Internet]. 2018;50(9):1219-24. Available from: https://doi.org/10.1038/s41588-018-0183-z

12. Khera A V, Chaffin M, Wade KH, Zahid S, Brancale J, Xia R, et al. Polygenic Prediction of Weight and Obesity Trajectories from Birth to Adulthood. Cell [Internet]. 2019 Apr 18;177(3):587-596.e9. Available from: https://doi.org/10.1016/j.cell.2019.03.028

13. Musliner KL, Mortensen PB, McGrath JJ, Suppli NP, Hougaard DM, BybjergGrauholm J, et al. Association of Polygenic Liabilities for Major Depression, Bipolar Disorder, and Schizophrenia With Risk for Depression in the Danish PopulationAssociation of Polygenic Liabilities With Risk for Depression in the Danish PopulationAssociation of Polygenic . JAMA Psychiatry [Internet]. 2019 May 1;76(5):516-25. Available from:

https://doi.org/10.1001/jamapsychiatry.2018.4166

14. Vassos E, Di Forti M, Coleman J, lyegbe C, Prata D, Euesden J, et al. An Examination of Polygenic Score Risk Prediction in Individuals With First-Episode 
Psychosis. Biol Psychiatry [Internet]. 2017 Mar;81(6):470-7. Available from: https://linkinghub.elsevier.com/retrieve/pii/S0006322316326646

15. Riglin L, Collishaw S, Richards A, Thapar AK, Maughan B, O’Donovan MC, et al. Schizophrenia risk alleles and neurodevelopmental outcomes in childhood: a population-based cohort study. The lancet Psychiatry [Internet]. 2017 Jan;4(1):57-62. Available from: http://www.ncbi.nlm.nih.gov/pubmed/27932233

16. Clarke T-K, Lupton MK, Fernandez-Pujals AM, Starr J, Davies G, Cox S, et al. Common polygenic risk for autism spectrum disorder (ASD) is associated with cognitive ability in the general population. Mol Psychiatry [Internet]. 2016 Mar;21(3):419-25. Available from: http://www.ncbi.nlm.nih.gov/pubmed/25754080

17. Zheutlin AB, Dennis J, Karlsson Linnér R, Moscati A, Restrepo N, Straub P, et al. Penetrance and Pleiotropy of Polygenic Risk Scores for Schizophrenia in 106,160 Patients Across Four Health Care Systems. Am J Psychiatry [Internet]. 2019 Aug 16;appi.ajp.2019.1. Available from:

http://ajp.psychiatryonline.org/doi/10.1176/appi.ajp.2019.18091085

18. Ruderfer DM, Ripke S, McQuillin A, Boocock J, Stahl EA, Pavlides JMW, et al. Genomic Dissection of Bipolar Disorder and Schizophrenia, Including 28 Subphenotypes. Cell [Internet]. 2018 Jun 14;173(7):1705-1715.e16. Available from: https://doi.org/10.1016/j.cell.2018.05.046

19. Brainstorm Consortium, Anttila V, Bulik-Sullivan B, Finucane HK, Walters RK, Bras J, et al. Analysis of shared heritability in common disorders of the brain. Science [Internet]. 2018;360(6395). Available from:

http://www.ncbi.nlm.nih.gov/pubmed/29930110

20. Lichtenstein P, Yip BH, Björk C, Pawitan Y, Cannon TD, Sullivan PF, et al. Common genetic determinants of schizophrenia and bipolar disorder in Swedish families: a population-based study. Lancet (London, England) [Internet]. 2009 Jan 17;373(9659):234-9. Available from:

http://www.ncbi.nlm.nih.gov/pubmed/19150704

21. Sartorious N. Comorbidity of mental and physical diseases: a main challenge for medicine of the 21st century. Shanghai Arch psychiatry [Internet]. 2013 Apr;25(2):68-9. Available from: http://www.ncbi.nlm.nih.gov/pubmed/24991137 
22. Verma A, Lucas A, Verma SS, Zhang Y, Josyula N, Khan A, et al. PheWAS and Beyond: The Landscape of Associations with Medical Diagnoses and Clinical Measures across 38,662 Individuals from Geisinger. Am J Hum Genet [Internet]. 2018 Apr 5;102(4):592-608. Available from:

https://doi.org/10.1016/j.ajhg.2018.02.017

23. Das S, Forer L, Schönherr S, Sidore C, Locke AE, Kwong A, et al. Nextgeneration genotype imputation service and methods. Nat Genet [Internet]. 2016;48(10):1284-7. Available from:

http://www.ncbi.nlm.nih.gov/pubmed/27571263

24. Galinsky KJ, Bhatia G, Loh P-R, Georgiev S, Mukherjee S, Patterson NJ, et al. Fast Principal-Component Analysis Reveals Convergent Evolution of ADH1B in Europe and East Asia. Am J Hum Genet [Internet]. 2016 Mar 3;98(3):456-72.

Available from: http://www.ncbi.nlm.nih.gov/pubmed/26924531

25. Chang CC, Chow CC, Tellier LC, Vattikuti S, Purcell SM, Lee JJ. Secondgeneration PLINK: rising to the challenge of larger and richer datasets.

Gigascience [Internet]. 2015;4:7. Available from:

http://www.ncbi.nlm.nih.gov/pubmed/25722852

26. Vilhjálmsson BJ, Yang J, Finucane HK, Gusev A, Lindström S, Ripke S, et al. Modeling Linkage Disequilibrium Increases Accuracy of Polygenic Risk Scores. Am J Hum Genet [Internet]. 2015 Oct 1;97(4):576-92. Available from: http://www.ncbi.nlm.nih.gov/pubmed/26430803

27. International HapMap 3 Consortium, Altshuler DM, Gibbs RA, Peltonen L, Altshuler DM, Gibbs RA, et al. Integrating common and rare genetic variation in diverse human populations. Nature [Internet]. 2010 Sep 2;467(7311):52-8.

Available from: http://www.ncbi.nlm.nih.gov/pubmed/20811451

28. Denny JC, Bastarache L, Ritchie MD, Carroll RJ, Zink R, Mosley JD, et al. Systematic comparison of phenome-wide association study of electronic medical record data and genome-wide association study data. Nat Biotechnol [Internet]. 2013 Dec;31(12):1102-10. Available from: http://www.ncbi.nlm.nih.gov/pubmed/24270849

29. Wu P, Gifford A, Meng X, Li X, Campbell H, Varley T, et al. Developing and 
Evaluating Mappings of ICD-10 and ICD-10-CM Codes to PheCodes. bioRxiv [Internet]. 2019 Jan 1;462077. Available from: http://biorxiv.org/content/early/2019/07/03/462077.abstract

30. Development Core Team R. R: A language and environment for statistical computing. R Foundation for Statistical Computing; 2018.

31. Sullivan PF, Agrawal A, Bulik CM, Andreassen OA, Børglum AD, Breen G, et al. Psychiatric Genomics: An Update and an Agenda. Am J Psychiatry [Internet]. 2017;175(1):15-27. Available from: https://doi.org/10.1176/appi.ajp.2017.17030283

32. Pomerleau OF, Downey KK, Stelson FW, Pomerleau CS. Cigarette smoking in adult patients diagnosed with attention deficit hyperactivity disorder. J Subst Abuse [Internet]. 1995;7(3):373-8. Available from:

http://www.ncbi.nlm.nih.gov/pubmed/8749796

33. Hübel C, Gaspar HA, Coleman JRI, Finucane H, Purves KL, Hanscombe KB, et al. Genomics of body fat percentage may contribute to sex bias in anorexia nervosa. Am J Med Genet B Neuropsychiatr Genet [Internet]. 2019 Sep;180(6):428-38. Available from:

http://www.ncbi.nlm.nih.gov/pubmed/30593698

34. Richards A, Horwood J, Boden J, Kennedy M, Sellers R, Riglin L, et al. Associations between schizophrenia genetic risk, anxiety disorders and manic/hypomanic episode in a longitudinal population cohort study. $\mathrm{Br} \mathrm{J}$ Psychiatry [Internet]. 2019;214(2):96-102. Available from:

http://www.ncbi.nlm.nih.gov/pubmed/30472973

35. Hall J. Schizophrenia - an anxiety disorder? Br J Psychiatry [Internet]. 2017 Nov;211(5):262-3. Available from: http://www.ncbi.nlm.nih.gov/pubmed/29092833

36. Cross-Disorder Group of the Psychiatric Genomics Consortium, Lee SH, Ripke S, Neale BM, Faraone S V, Purcell SM, et al. Genetic relationship between five psychiatric disorders estimated from genome-wide SNPs. Nat Genet [Internet]. 2013 Sep;45(9):984-94. Available from: http://www.ncbi.nlm.nih.gov/pubmed/23933821

37. Centers for Disease Control and Prevention. Tobacco-Related Disparities 
bioRxiv preprint doi: https://doi.org/10.1101/858027; this version posted November 29, 2019. The copyright holder for this preprint (which was not certified by peer review) is the author/funder, who has granted bioRxiv a license to display the preprint in perpetuity. It is made available under aCC-BY-NC-ND 4.0 International license.

[Internet]. 2018. Available from: https://www.cdc.gov/tobacco/disparities/index.htm 